5 WANG, T.H., and гтон, Т.: 'Compact grating structure for application to filters and resonators in monolithic microwave integrated circuits', IEEE Trans., 1987, MTT-35, pp. 1176-1182

6 BÖTTCHER, E.H., PFITZENMAIER, H., DRÖGE, F, and BIMBERG, D.: 'Millimetre-wave coplanar waveguide transmission lines on InP', Electron. Lett., 1996, 32, pp. 1377-1378

7 GÖUUR, A., KARPUZ, C., and ALKAN, M.: 'Characteristics of periodically loaded CPW structures', IEEE Microw. Guid. Wave Lett., 1998, 8, pp. 278-280

8 PetTenPaul, E., KAPUSTA, H., Weisgerber, A., MAMPE, H., LUGINSLAND, J., and WOLFF, I.: 'CAD models of lumped elements on GaAs up to $18 \mathrm{GHz}$ ', IEEE Trans., 1988, MTT-36, pp. 294-304

\section{Automatic self-matching network for industrial microwave heating based on conjugate gradient algorithm}

\section{J.J. Mallorquí, A. Aguasca, A. Cardama, R. Pagès and J. M ${ }^{\text {a }}$ Haro}

An automatic matching network has been implemented using a waveguide magic $\mathrm{T}$ with two sliding short circuit stubs which are adjusted by a microcontroller running a conjugate gradient algorithm. The inclusion of this device in an industrial heating system allows high energetic efficiency of the oven to be maintained despite changes in the load.

Introduction: One of the main problems regarding the efficiency of industrial heating systems using high power microwaves is related to uncontrolled changes in the load. These changes can be caused both by the different kinds of materials to be processed in the same oven and by the temperature rise which modifies the material's physical properties, particularly its dielectric permittivity. Usually, the microwave generator is matched to the oven by means of a waveguide matching system, typically a two, three or four capacitive-screw tuner empirically adjusted for a given load [1]. Once the heating process is started, the temperature dependence of the complex permittivity usually changes the impedance seen by the generator, worsening the mismatch factor and reducing the efficiency of the heating process. A similar problem affects an oven with inhomogeneous and discontinuous loads; it is not feasible to manually readjust the matching network for every different sample processed. Until now the solution has been simple but highly inefficient: to increase the incident power in order to overcome the mismatching effects. In this Letter we describe an autonomous system which is able to detect load mismatches and reduce them automatically.

Magic $T$ as matching network: The magic $\mathrm{T}$ is the waveguide implementation of a $180^{\circ}$ hybrid junction, a four-port network which has a $180^{\circ}$ phase shift between the two output ports [2]. Considering an ideal magic $\mathrm{T}$ with sliding shorts at the $E$ and $H$ planes, the input reflection coefficient $\Gamma_{\text {in }}$ can be expressed as a function of the load reflection coefficient $\Gamma_{L}$ and the electrical phases corresponding to the positions of the two sliding shorts, $\Gamma_{E}$ $=e^{j \Phi E}$ and $\Gamma_{H}=e^{j \oplus H}$ :

$$
\Gamma_{i n}=\frac{1}{2}\left(\frac{2\left(\Gamma_{E}+\Gamma_{H}\right)-4 \Gamma_{L} \Gamma_{E} \Gamma_{H}}{2-\left(\Gamma_{E}+\Gamma_{H}\right) \Gamma_{L}}\right)
$$

Several stub positions that match a given lossy load can be found, the number depending on their electrical length. Despite this simple relationship between $\Gamma_{L}$ and the stub positions, it is not easy to implement in an industrial microwave oven because it requires knowledge of both the magnitude and phase of $\Gamma_{L}$. A high power vector network analyser would need to be implemented and included in the oven. In addition, the working frequency of an industrial magnetron depends on the load, the so-called frequency pulling [3], and even when the load does not change, the magnetron's frequency stability is poor. With highly reactive loads the bandwidth of the matching network is narrow, so small frequency deviations could cause a large mismatch, even with a constant load. Therefore, a dynamic matching network able to react to both load and frequency changes is needed. The last practical aspect, but not the least important, is that even when the correct position of the stubs can be computed, during its positioning a temporal worsening of the mismatch losses will be produced.

Algorithm: The algorithm is based on the well-known conjugate gradient method (CGM) used to iteratively minimise functions which depend on one or more independent variables [4]. At each step the algorithm performs a linear minimisation of the function along a direction computed by using the local gradient and all the previous directions traversed. For quadratic functions, the procedure generates a set of mutually conjugated directions that, correctly weighted by the algorithm, reach the function minima. The number of required steps is equal to (or less than) the number of independent variables of the function or, in other words, the number of independent eigenvalues of the Hessian matrix used to define the quadratic function. Unfortunately, in the real world, functions representing real processes usually are not quadratic. Despite this, in most cases the CGM can still be used because functions usually exhibit quasi-quadratic behaviour near minima. In such cases, the number of iterations required to obtain the minima will be larger than the number of independent variables and will depend on both the kind of function and the starting point. When the function has different local minima the CGM does not require a global minimum.

The benefit of using CGM is that it only requires function evaluations, and not the algebraic knowledge of the function to be minimised. The required gradient evaluations can be computed using a typical finite difference scheme. In this application the initial problem has been reduced to the minimisation of the unknown near quadratic function, $\left|\Gamma_{i n}\right|=f\left(\Gamma_{L}, \Gamma_{E}, \Gamma_{H}\right)$, by means of successive function evaluations in a conjugate gradient scheme. The function has no local minima, but many identical minima due to the $\lambda / 2$ periodicity of the reflection coefficient.

Hardware: Two short circuits have been implemented in a circular waveguide, the dimensions of which are close to the cutoff frequency of the $T E_{11}$ mode in order to reduce its wavelength to a minimum. The short plungers have been designed using a folded half-wavelength capacitance coupling [5]. The measurement of the reflection coefficient inside the rectangular waveguide has been implemented with a double directional coupler using a hybrid technology (waveguide-microstrip) which minimises its volume [6]. Two power detectors at the coupled branches provide a voltage proportional to the power carried by the incident and reflected waves. Finally, one microcontroller, a Microchip PIC16C74, runs the CGM with the measurements and controls the stub positions by means of two stepper motors. The experimental prototype can be seen in Fig. 1.

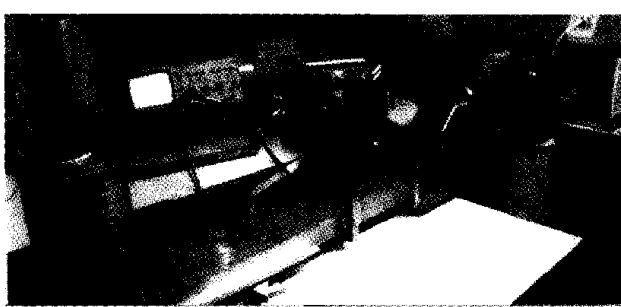

Fig. 1 Experimental prototype

From background to foreground: magnetron, magic $\mathrm{T}$ with two cylindrical stubs, box with microcontroller and auxiliary electronics, and cavity

Results: The system has shown its ability to automatically match changing loads inside an industrial microwave oven. Among the different tests carried out, the drying of a wet sponge is presented to show the typical behaviour of a changing load inside a small cavity. Fig. $2 a$ shows the temporal evolution of the input reflection coefficient depending on the matching network used. When a manual three-screw tuner is used it is difficult to achieve more than $10 \mathrm{~dB}$ and, once the water starts to evaporate, the mismatching increases, leading to an oscillatory behaviour. If the automatic matching network is placed between the magnetron and the load, the CGM scheme is able to maintain an average return loss better than $15 \mathrm{~dB}$. The benefits of the automatic matching network are 
evident at the end of the drying process, when the reduced levels of water inside the sponge make the load more reactive. For extremely reactive loads the stub positions which match a given load are more critical; then small changes in the magnitude or phase of its reflection coefficient will cause large changes in the reflection coefficient seen by the magnetron, as shown by the oscillatory behaviour of the $\Gamma_{\text {in }}$ (automatic network) plot. Fig. $2 b$ presents the energetic efficiency for both cases in terms of the power transfer percentage. While the automatic network ensures an efficiency of $>95 \%$ for most of the drying process, the efficiency of the manual system only stays at $\sim 75 \%$, falling to $25 \%$ at the end of the process. The automatic system is able to match extremely reactive loads. One of the tests consisted in matching an empty cavity, and it was found that it was possible to dissipate practically all the available power of the magnetron in the metal walls of the cavity. As a result, a significant temperature increase could be observed in the oven walls.
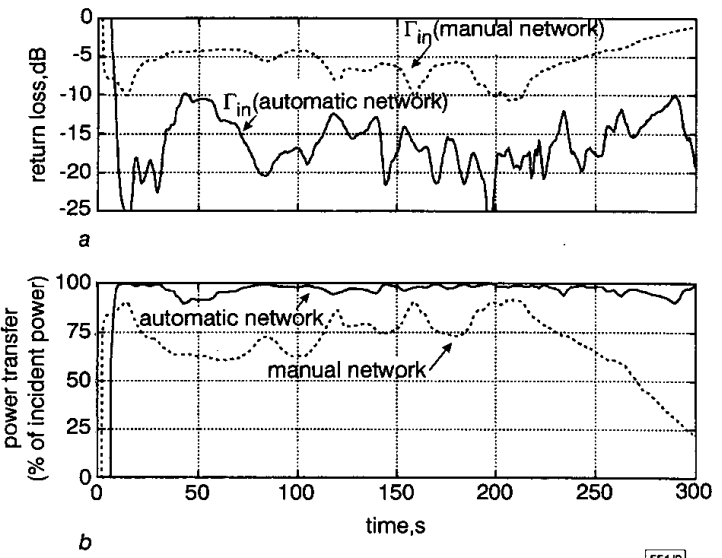

$551 / 2$

Fig. 2 Comparison between temporal behaviour of return loss in manual and automatic systems, and transferred power from magnetron to load

a Comparison between temporal behaviour of return loss when changing load is manually adjusted with three screw tuner, $\Gamma_{\text {in }}$ (manual network), and when automatic magic $T$ is used, $\Gamma_{\text {in }}^{\text {in }}$ (automatic network)

$b$ Transferred power from magnetron to load for both cases

Conclusions: An automatic matching network for microwave industrial applications based on a conjugate gradient scheme has been presented. The combination of a waveguide $180^{\circ}$ hybrid junction with two sliding short circuits controlled by a microcontroller has shown the potential capability to increase the efficiency of industrial processes involving unknown or changing highly reactive loads in microwave ovens.

Acknowledgments: This work was sponsored by the company CIM-D'OR and the Generalitat de Catalunya under grant CIRITCIDEM. We would like also to thank A. Cano for his help and advice on the design and construction of the different mechanical parts.

(ㄷ) IEE 1999

Electronics Letters Online No: 19990219

5 January 1999 DOI: $10.1049 /$ l: 19990219

J.J. Mallorquí, A. Aguasca, A. Cardama, R. Pagès and J. M ${ }^{\mathrm{a}}$ Haro (Department of Signal Theory and Communications, Universitat Politècnica de Catalunya, Campus Nord UPC-D3, C/ Jordi Girona 1-3, 08034 Barcelona, Spain)

E-mail: mallorqu@voltor.upc.es

\section{References}

1 ROUSSY, G., and PIERCE, J.A.: 'Foundations and industrial applications of microwave and radio frequency fields' (John Wiley \& Sons, 1995)

2 POZAR, D.M.: 'Microwave engineering' (Addison-Wesley Publishing Co., 1993)

3 GILMOUR, A.S., Jr.: 'Microwave tubes' (Artech House, 1986)

4 PRESS, W.H., and FLANNERY, B.P.: 'Numerical recipes in C: The art of scientific computing' (Cambridge University Press, 1989)
5 RAGAN GI. 'Microwave transmission circuits' (Dover Publications, Inc. 1968)

6 CARON, M., AKYEL, C., and GHANNOUCHI, F.M. 'A versatile easy to do six-port based high power reflectometer', J. Microw. Power Electromagn. Energy, 1995, 30, (4), pp. 231-239 (International Microwave Power Institute)

\section{Improving FBF neurofuzzy approximator by optimised input space covering}

\author{
A. Mancini, F.M. Frattale Mascioli, A. Rizzi and \\ G. Martinelli
}

Possible improvements to neurofuzzy nets based on the Mamdani model are discussed. A new algorithm using an optimised clustering technique is proposed and a comparative test presented.

Introduction: A large number of neurofuzzy networks are based on the classical Mamdani model. Particularly interesting in this regard is the FBF (fuzzy basis function) approach [1]. This interprets the net output as the expansion of the unknown mapping in a suitable basis of functions, which are related to rules extracted by numerical and linguistic data. Several alternatives are possible depending on the choice of the basic ingredients of the Mamdani model (fuzzy logic reasoning, membership functions, fuzzy operations, type of fuzzification/defuzzification). Consequently, in spite of the widespread use of neurofuzzy nets in actual applications, there still exists the necessity for improving their performance. A clear example is presented in [2], where a simple modification of classical fuzzy logic reasoning is introduced by using a compensatory parameter, with the effect that the resulting mapping is significantly improved.

In this Letter, we focus our attention on FBF implementation. We show how optimised input space coverage is able to greatly improve net performance. We propose an efficient method for tailoring the membership functions (MFs) of the rules, extracted from the numerical data. The method is based on an optimised clustering of data in the conjunct input-output space.

Network architecture: The FBF net relies on the Mamdani model, which is based on IF-THEN rules where both the antecedent and the subsequent parts are fuzzy quantities. Suppose that $N$ rules have been extracted from the available data; the $i$ th rule has the following structure:

$$
\text { if } x_{1} \text { is } A_{1}^{(i)} \text { and...and } x_{n} \text { is } A_{n}^{(i)} \text {, then } y \text { is } B^{(i)}
$$

where $A_{k}^{(i)}$ and $B^{(i)}$ are fuzzy sets and $n$ is the input space dimension. Several alternative versions of the FBF nets can be considered depending on the choice of MF, the type of fuzzifier/ defuzzifier, and the $t$-norm to be used in the manipulation of the fuzzy quantities. In the following, we will adopt the same options considered in [1]. Consequently, the net output is given by

$$
y=\sum_{i=1}^{N} y^{(i)} \alpha^{(i)}(\underline{x})
$$

where $\underline{x}$ is the input, i.e. $\underline{x}=\left[x_{1}, \ldots, x_{n}\right], \alpha^{(i)}(\underline{x})$ is the ith PBF, and $y^{(i)}$ is the centroid of the MF of $B^{(i)}$. The structure of $\alpha^{(i)}(\underline{x})$ is

$$
\alpha^{(i)}(\underline{x})=\frac{\prod_{k=1}^{n} \mu_{A_{k}^{(i)}}\left(x_{k}\right)}{\sum_{j=1}^{N}\left[\prod_{k=1}^{n} \mu_{A_{k}^{(j)}}\left(x_{k}\right)\right]}
$$

where $\mu_{A_{k}(i)}\left(x_{k}\right)$ is the MF of the input $x_{k}$ referred to the $i$ th rule:

$$
\mu_{A_{k}^{(i)}}\left(x_{k}\right)=\exp \left[-1 / 2\left(x_{k}-x_{k}^{(i)}\right)^{2} / \sigma_{k}^{(i)^{2}}\right]
$$

Proposed algorithm: To optimise the MFs of the rules, it is necessary to extract them from numerical data. The MFs should be able to cover the input space with specific reference to the training set distribution. This goal can be attained by clustering the exam- 